\title{
COMBINED LONGITUDINAL AND TRANSVERSE MULTITURN INJECTION IN A HEAVY ION ACCUMULATOR
}

\author{
Ch. Carli, S. Maury, D. Möhl, CERN/PS, CH 1211 Geneva 23, Switzerland
}

\begin{abstract}
After the completion of the antiproton programme, the Low-Energy Antiproton Ring (LEAR) will be able to serve as an accumulator ring for heavy ions in the injector chain of the Large Hadron Collider (LHC). For injection and accumulation, a scheme is proposed, which exploits both the longitudinal and the transverse acceptances of LEAR. Compared to a classical multiturn injection, a factor 3 to 5 in intensity is gained for the set of parameters pertaining to this case; furthermore emittances, which are more favorable for electron cooling, result. The principle is outlined and computer simulations are presented.
\end{abstract}

\section{INTRODUCTION}

To prepare intense bunches of ions for injection into the LHC, it has been proposed [1] to cool and accumulate heavy ions in LEAR or a similar ring constructed for this purpose. Particles coming from the linac are injected by multiturn injection and cooled and stacked by electron cooling; the process is repeated until the required intensity is reached. In order to increase the number of injected particles and to obtain a particle distribution more favorable for electron cooling, an injection scheme has been proposed [2], which exploits both the longitudinal and the transverse acceptances.

Computer simulations to investigate this new technique of combined longitudinal and transverse injection for LEAR have been performed. Some results will be presented here. A more detailed description of the simulations can be found in [3]. A summary of recent experiments in view of ion accumulation is given in [4].

\section{PRINCIPLE}

The principle of combined longitudinal and transverse multiturn injection is best explained with the help of Fig. 1. As for convential multiturn injection, a local deformation of the closed-orbit (the"bump") is created and decreases during the injection of the linac pulse. The linac energy is ramped in such a manner, that at the injection septum the closed-orbit corresponding to the instantaneous linac energy remains fixed, or changes only by a small amount.

For conventional transverse multiturn injection, the linac energy is constant and the closed-orbit moves away from the septum as the bump decreases. Thus a large emittance of the circulating beam results whereas the momentum spread is ideally the same as that of the incoming beam. With the combined injection (which has some similarities with the "painting" used in $\mathrm{H}^{-}$injection [5]) both longitudinal and transverse phase space are exploited and transverse emittance can be traded for momentum spread.

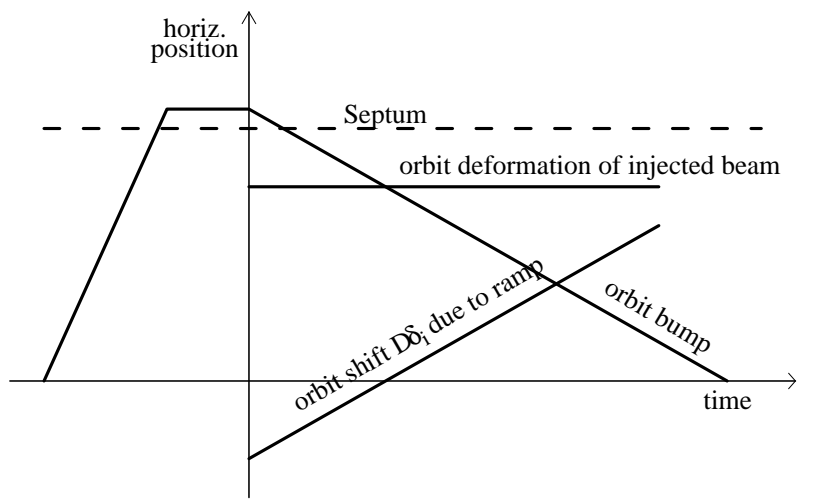

Figure 1: Horizontal positions for an example of combined longitudinal and transverse multiturn injection. The closedorbit bump decreases during the injection of the pulse as for conventional multiturn injection. The momentum of the incoming beam is ramped $p=p_{0}\left(1+\delta_{i}(t)\right)$, which leads to an additional time dependent orbit shift $D \delta_{i}(t)$ (where $D$ is the dispersion) for the incoming beam. The bump decrease and the energy ramp are adjusted, such that the instantaneous closed-orbit of the injected particles remains fixed.

\section{LEAR LATTICES}

With the fixed geometry of LEAR different "machines" can be obtained varying the currents in the quadrupole families. For the injection a small horizontal $\beta$ function and a large dispersion are necessary; whereas for the electron cooling we expect vanishingly small dispersion and intermediate $\beta$ in both transverse phase spaces to be advantageous. In Fig. 2, the Twiss functions are shown for two of the machines used for the simulations; (a) shows standard LEAR and (b) the machine best for combined injection, but with $\beta$ functions a little too large for electron cooling. A third lattice used for the simulations has smaller $\beta$-functions at the cooler, but leads to a situation less suitable for the injection.

\section{SIMULATIONS}

The injection process is simulated with a Monte-Carlo method. Each particle is described by its coordinates in the two transverse phase spaces, the relative momentum deviation and the time. First a Gaussian beam with particle coordinates corresponding to given Twiss parameters is gener- 


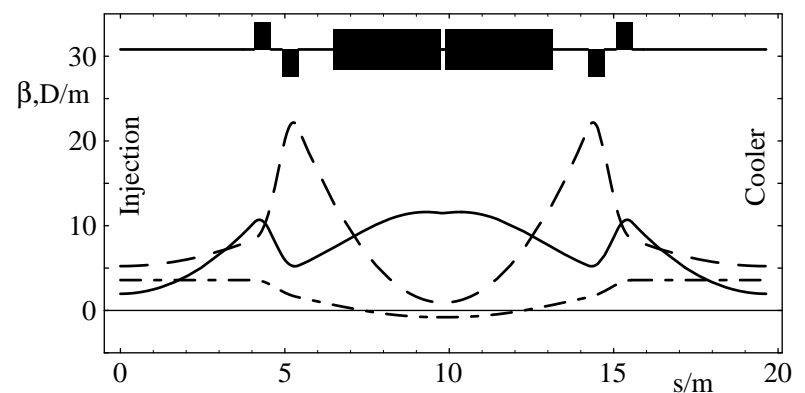

a)

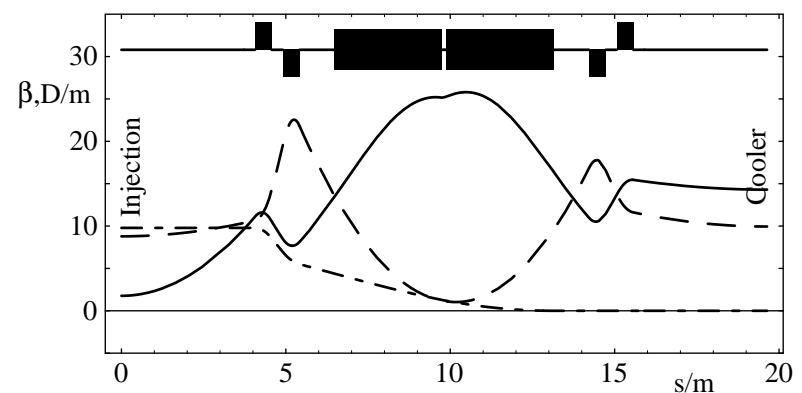

b)

Figure 2: Lattice functions (the solid and dashed lines represent the horizontal and vertical $\beta$-function respectively, the dot-dashed line is for the dispersion) for one fourth of the machine extending from the center of the injection to the center of the electron cooling straight section for (a) standard LEAR and (b) a lattice improved for combined multiturn injection. The lattice functions in the rest of the accelerator are obtained by mirroring and repetition.

ated at the injection septum with a random number generator. The injected particles are tracked through the accelerator consisting of the lattice magnets (dipoles, quadrupoles), closed-orbit bumpers, aperture limitations and the injection septum. Particles hitting an aperture limitation or the septum or falling outside an acceptance of $50 \mu \mathrm{m}$ at the end of the injection are counted as lost. In simulations of a purely transverse process, the transverse acceptance had to be increased to $150 \mu \mathrm{m}$ to permit injection of a sufficient number of turns.

For the beam coming from the linac the following characteristics (corresponding to one standard deviation) were taken from [6] :

emittance $\quad: 2.1 \mu \mathrm{m}$

relative momentum spread : $0.210^{-3}$

In addition to the (instantaneous) spread, the mean momentum of the linac beam changes with time; this ramp has been limited to $\delta<610^{-3}$. Further it is required that the distance from the center of the stack to the vacuum chamber must be at least 2.5 times the standard deviation of the stack. This leads to the following phase space volumes $(2.5 \mathrm{rms})$ reserved for the stack (volumes expected for $1 \mathrm{rms}$ taken from [1]) : horizontal emittance $\quad: \quad(2.5)^{2} \cdot 10 \mu \mathrm{m}=62.5 \mu \mathrm{m}$ vertical emittance $\quad: \quad(2.5)^{2} \cdot 5 \mu \mathrm{m}=31.2 \mu \mathrm{m}$ rel. momentum spread $\quad: \quad 2.5 \cdot 0.510^{-3}=1.2510^{-3}$ where the contributions due to momentum spread and due to horizontal betatron oscillations have to be added quadratically.

For the model of the accelerator, the real apertures available were followed as close as possible, after removing $10 \mathrm{~mm}$ in order to account for closed-orbit distortions up to $\pm 5 \mathrm{~mm}$.

To optimize the injection efficiency all parameters of the incoming beam (position, angle, Twiss parameters, momentum) and the position of the injection septum are varied. The mean momentum of the stack is such that it is near the inner wall of the vacuum chamber, whereas the injection is from outside.

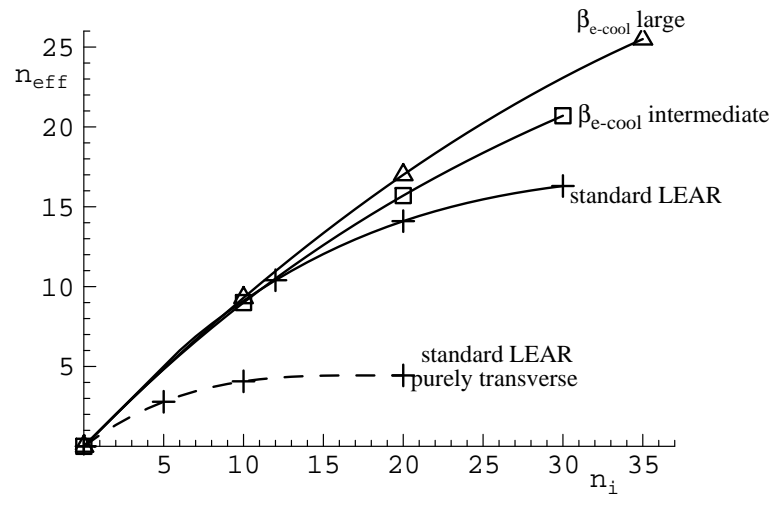

Figure 3: Number of 'efficient' turns $n_{\text {eff }}$ accepted per injection as a function of the number of injected turns $n_{i}$ for optimizations of combined multiturn injection. The crosses are for standard LEAR; triangles and squares are for injection into improved lattices with large and intermediate betatron functions at the electron cooler. For comparision, efficiencies for a purely transverse process and standard LEAR machine are given as a dashed line. In the latter case the full acceptance $(150 \mu \mathrm{m})$ of LEAR is used whereas in all other cases the emittance was limited to $50 \mu \mathrm{m}$ to permit faster cooling.

In Fig. 3 the number of turns accepted as a function of injected turns is plotted for various conditions. Phase-space plots for injection of 20 turns into the standard LEAR machine and into the machine best suited for combined injection (but expected to be less appropriate for electron cooling) are given in Figs. 4 and 5. For the incoming beam three phase space ellipses with small momentum deviations were overlapped; they separate after injection due to the dispersion. One notes that this separation is more important for the improved lattice because the normalized Dispersion $d / \sqrt{\beta}$ at the injection septum is increased; this is a consequence of improving the lattice for combined injection with a limited linac energy ramp.

For comparision, a plot of the horizontal phase space for the purely transverse process and the standard LEAR 

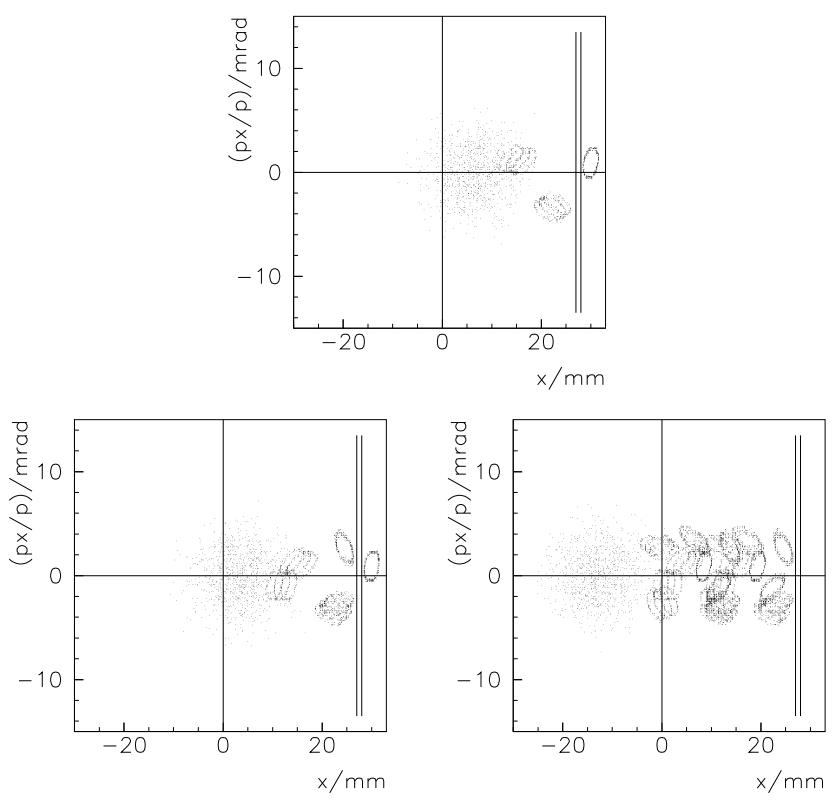

Figure 4: Plots of the horizontal phase space for the combined longitudinal transverse multiturn injection into the standard LEAR machine. 20 turns are injected; the three plots show the particles after 2, 5 and 20 turns.
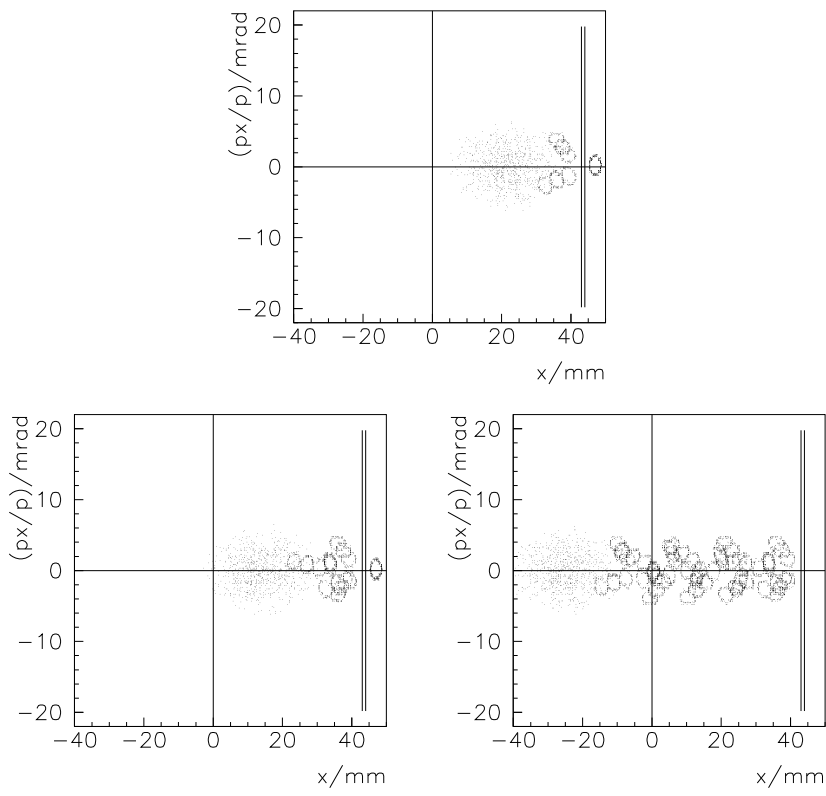

Figure 5: Plots of the horizontal phase space for the combined longitudinal transverse multiturn injection into the improved lattice with large $\beta$ at the cooler. 20 turns are injected; the three plots show the particles after 2, 5 and 20 turns.

machine is given in Fig. 6. One notes, that particles are injected only in a ring-shaped region of the phase space around the stack.
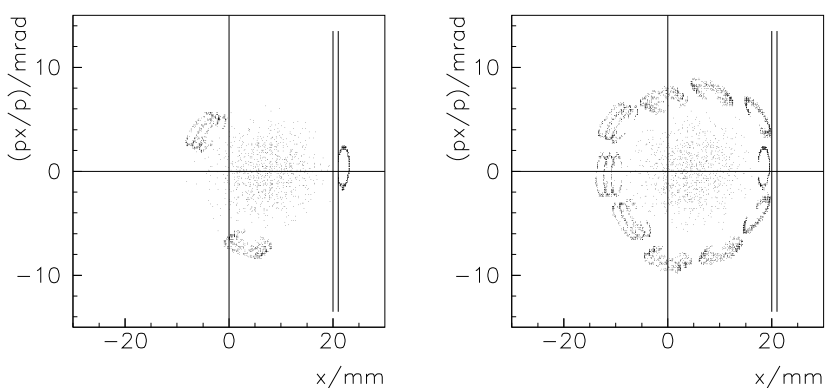

Figure 6: Plots of the horizontal phase space for purely transverse multiturn injection into the standard LEAR machine. 10 turns are injected; the two plots show the particles after 2 and 10 turns.

\section{CONCLUSIONS}

Comparing the number of turns that can be injected with a given efficiency, one notes that a factor of 3 to 5 is gained with combined injection as compared to the purely transverse process (in the standard LEAR machine); another factor of 2 can be gained if the lattice is modified. In addition a smaller emittance of the injected beam, more favorable for electron cooling, is possible with the combined injection.

\section{REFERENCES}

[1] P. Lefèvre and D. Möhl, A low Energy Accumulation Ring of Ions for LHC, CERN/PS 93-62 and LHC Note 259.

[2] S. Maury and D. Möhl, Combined Longitudinal and Transverse Multiturn Injection into a Heavy Ion Accumulator Ring, internal note (CERN) PS/AR/Note 94-12.

[3] Ch. Carli, Simulation of Multiturn Injection into LEAR in View of Ion Accumulation, internal note (CERN) PS/DI/Note 96-24.

[4] J. Bosser, J. Broere, C. Carli, M. Chanel, C. Hill, R. Ley, A. Lombardi, R. Maccaferri, S. Maury, D. Möhl, G. Molinari, H. Mulder, E. Tanke, G. Tranquille, M. Vretenar, Recent Lead Ion Storage Tests on LEAR, this conference.

[5] D. Raparia, C.W. Planner, G.H. Mackenzie and J.R. Richardson, Efficient Capture in an Accumulator, of 20000 Turns of Beam Injected from TRUMF, Proc. of PAC 1985 (Vancouver), IEEE Trans. Nucl. Sci. NS-32, p 2456.

[6] N. Angert, M.P. Bourgarel, E. Brouzet, R. Cappi, D. Dekkers, J. Evans, G. Gelato, H. Haseroth, C.E. Hill, G. Hutter, J. Knott, H. Kugler, A. Lombardi, H. Lustig, E. Malwitz, F. Nitsch, G. Parisi, A. Pisent, U. Raich, U. Ratzinger, L. Riccati, A. Schempp, K. Schindl, H. Schönauer, P. Têtu, H.H Umstätter, M. van Rooij, D. Warner and M. Weiss, CERN Heavy-Ion Facility Design Report, CERN 93-01.

and

N. Angerth, W. Bleuel, Y. Bylinsky, H. Gaiser, H. Haseroth, G. Hutter, H. Kugler, E. Malwitz, R. Popescu, M. Rau, U. Ratzinger, R. Scrivens, E. Tanke, D. Warner, The IH Linac of the CERN Lead Injector, proceedings of the 1994 international linac conference, Tsukuba, Japan 